\title{
On the Average Pairwise Connectivity of Wireless Multihop Networks
}

\author{
Fangting Sun and Mark Shayman \\ Department of Electrical and Computer Engineering \\ University of Maryland, College Park, MD 20742 \\ \{ftsun, shayman\}@eng.umd.edu
}

\begin{abstract}
A new metric-average pairwise connectivity-is proposed to measure the fault tolerance of wireless multihop networks. Two types of random graphs, Poisson random graph and geometric random graph, are adopted to model wireless multihop networks and are used in experimental studies. By investigating the upper bound and lower bound of the average pairwise connectivity for different types of random graphs and the distribution of the difference between the upper bounds and lower bounds, we conclude that the average pairwise connectivity of wireless multihop networks can be very well approximated by its upper bound, a quantity that is easily computed.
\end{abstract}

\section{INTRODUCTION}

Wireless multihop networks are formed by groups of nodes connected by wireless links in which the nodes can communicate with other nodes beyond their direct transmission range by cooperatively forwarding packets for each other. Examples of such networks include wireless ad hoc networks and sensor networks. Since wireless multihop networks can be easily and inexpensively set up as needed in a decentralized manner, they have a wide range of applications.

However, before wireless multihop networks can be successfully deployed, reliability issues must be resolved. In this paper we investigate one fundamental property of wireless multihop networks, namely network fault tolerance. Roughly speaking, network fault tolerance denotes the ability of a network to continue to operate correctly even though some of its components have malfunctioned or failed. Important sources of failure include link breakage and node removal. Link breakage may happen very frequently due to the fragile wireless connections or possible mobility, and nodes may be removed from the network due to exhaustion of battery power. Consequently, the study of fault tolerance should be an indispensable component when designing and evaluating such networks.

In this paper, we focus on the quantification of fault tolerance for networks, specifically, wireless multihop networks. Traditionally, network fault tolerance has been expressed as the network's connectivity. For example, network fault tolerance has been defined as the maximum number of elements that can fail without inducing a possible disconnection in the network [1]. However, such measures have not accounted for the total number of nodes in the network and the probability of a

This research was partially supported by AFOSR under grant F496200210217. disconnection. To overcome the limitation of such measures, a probabilistic measure of network fault tolerance based on the disconnection probability was proposed in [2]. The fault tolerance of a family of regular graph network topologies have been evaluated based on this measure, and the results show that the overall disconnection probability in such networks can be approximated by the probability of a single node disconnection.

Recently, the fault tolerance of wireless multihop networks has drawn much attention from academia, military, and industry due to the wide range of potential applications and the inherent fragility of such networks. In [3], the minimum node degree and $k$-connectivity of wireless multihop networks has been studied, where a wireless multihop network is modeled as a geometric random graph [4]. Similar results to [2] have also been obtained; with a very high probability the connectivity of wireless multihop network is equal to the minimum node degree among all nodes in the network.

The use of $k$-connectivity as a metric for network fault tolerance focuses on the worst case. It implies that there is some choice of $k+1$ nodes whose removal would disconnect the network. However, that does not mean that if $k+1$ nodes are removed, it is likely that the network will be disconnected. Furthermore, even if the removal of a group of nodes disconnects the network, it is possible that only one node becomes isolated from the rest. This may not have a significant impact on the usefulness of the network. Consequently, instead of considering fault tolerance from a worst case perspective, we consider it from the viewpoint of average behavior. Specifically, we propose a new measure to evaluate the fault tolerance of wireless multihop networks: the average pairwise connectivity which is defined as the average connectivity degree among all pairs of nodes in the network. We use acronym APC to represent the average pairwise connectivity in the rest of paper.

In this paper we model the wireless multihop networks using random graphs. Two general random graph models have been studied: Poisson random graphs [5], [6] and geometric random graphs [7]. Each can be used to model different scenarios. We investigate the upper bound and lower bound of the APC for these two types of random graphs. The difference between the upper bounds and lower bounds is also studied, and our results show that APC of these types of networks can be approximated very well using their upper bounds. 
Furthermore, the estimation expression for the upper bound of APC is derived, and is easily computed.

The rest of this paper is organized as follows. Section II defines the proposed metric to measure the fault tolerance of wireless multihop networks. Section III describes the different types of random graphs used to model the wireless multihop networks. Section IV investigates the fault tolerance of different types of random graphs through experimental studies. Finally, Section V concludes this paper.

\section{Average Pairwise Connectivity}

In this section, we first clarify the notations for graphs, then define average pairwise connectivity.

An undirected graph $G=G(V, E)$ comprises $|V|$ nodes and $|E|$ edges, and for any $u, v \in V$, if $(u, v) \in E$, then $(v, u) \in E$. For $u \in V$, let $d(u)$ denote the degree of $u$, the number of neighbors of node $u$. We say two nodes $u$ and $v$ are connected if there exists at least one path between $u$ and $v$; otherwise these two nodes are said to be disconnected. A set of paths from $u$ to $v$ are said to be node-disjoint if these paths do not share any common nodes except $u$ and $v$. For any pair of nodes $u, v \in V$, let $C(u, v)$ denote the maximum number of node-disjoint paths from node $u$ to node $v$. Since $G$ is undirected, $C(u, v)=C(v, u)$. In this paper, $C(u, v)$ is referred to as the pairwise connectivity between $u$ and $v$. If $C(u, v)=k$, there exist no such set of $k-1$ nodes whose removal would make $u$ and $v$ disconnected. We say that the node pair $(u, v)$ is $k$-pairwise-connected if and only if $C(u, v) \geq k$. The graph $G$ is said to be connected if any pair of nodes in $G$ is connected. Moreover, the graph $G$ is said to be $k$-connected if for any pair of nodes $u, v \in V, C(u, v) \geq k$ [4].

To measure the fault tolerance of networks, in this paper we propose to use the metric: average pairwise connectivity. Given an undirected graph $G(V, E)$, the APC of $G$, denoted by $C(G)$, is defined as follows:

$$
C(G)=\frac{1}{N(N-1)} \sum_{u \in V} \sum_{v \neq u \in V} C(u, v) .
$$

\section{NETWORK MODELS}

The theory of random graphs uses probabilistic methods to solve problems in graph theory. The Poisson random graph, which is independently proposed by Solomonoff and Rapoport [8], and Erdos and Renyi [5], [6], is one of the simplest and most useful models in random graphs. In [9], Chlamtac and Faragó suggest modeling ad hoc networks as Poisson random graphs. However, Poisson random graphs do not take into account correlations between different links. To fix this problem, the geometric random graph is proposed to model ad hoc networks. In this paper, we will study the APC metric in both the Poisson random graph model and geometric random graph model.

\section{A. Poisson random graph}

The Poisson random graph is perhaps the simplest and most useful model for a wireless network, which has been well studied by mathematicians, and many results, both approximate and exact, have been proved [10], [11]. Since the Poisson random graph model has many elegant properties, it has been widely used in network modeling performance analysis.

A Poisson random graph $G(N, p)$ is a graph with $N$ nodes in which for each pair of nodes, with probability $p$ there is an edge between them. By holding the mean degree $\lambda=p(N-1)$ constant, the probability of a node having degree $k$ can be calculated as

$$
p_{k}=\left(\begin{array}{c}
N-1 \\
k
\end{array}\right) p^{k}(1-p)^{N-1-k} \simeq \frac{e^{-\lambda} \lambda^{k}}{k !},
$$

with the last approximate equality becoming exact in the limit of large $N$ and fixed $k$, from which the name "Poisson random graph" comes.

\section{B. Geometric random graph}

As another simple network model, the geometric random graph has also been used to model various kinds of networks. For example, it has been widely used to model the topology distribution of wireless ad hoc networks [3]. A geometric random graph $G(N, r)$ is a graph in which $N$ nodes are independently deployed inside a large area of size $A$ according to the $2 \mathrm{D}$ uniform distribution, and for any pair of nodes there exists an edge between these two nodes if and only if the distance between them is no more than $r$ (e.g., in wireless multihop networks, $r$ is nodes' maximum transmission range). By holding $\lambda=\frac{(N-1) \pi r^{2}}{A}$ to be constant, the probability of a node having degree $k$ can be calculated as

$p_{k}=\left(\begin{array}{c}N-1 \\ k\end{array}\right)\left(\frac{\pi r^{2}}{A}\right)^{k}\left(1-\frac{\pi r^{2}}{A}\right)^{N-1-k} \simeq \frac{e^{-\lambda} \lambda^{k}}{k !}$,

with the last approximate equality becoming exact in the limit of large $A$ and $N$ and fixed $k$. That is, the distribution of degree also follows the Poisson distribution with the mean degree being $\lambda$.

\section{EXPERIMENTAL RESUlts AND Discussion}

In this section we will focus on the experimental studies of the APC in two different network models. In the experiments, for any given pair of nodes $(u, v)$, we compute an upper bound $C_{\text {upper }}(u, v)$ and lower bound $C_{\text {lower }}(u, v)$.

For a given graph $G(V, E)$ and $u, v \in V$, the number of node-disjoint paths between $u$ and $v$ cannot exceed the minimum degree of $u$ and $v$, so the upper bound $C_{u p p e r}(u, v)$ is defined as follows:

$$
C_{\text {upper }}(u, v)=\min \{d(u), d(v)\} .
$$

To find the lower bound $C_{\text {lower }}(u, v)$, the following method is used. We initialize $C_{\text {lower }}(u, v)$ to be 0 , and initialize graph $G_{0}$ to be $G$, then repeat the following steps: at each step we try to find the shortest path between $u$ and $v$, if there is no such path, the procedure terminates; otherwise, remove all the intermediate nodes belonging to this path as well as all the edges directly connected with those nodes from $G_{C_{l o w e r}(u, v)}$, and increase the value of $C_{\text {lower }}(u, v)$ by 1 . 

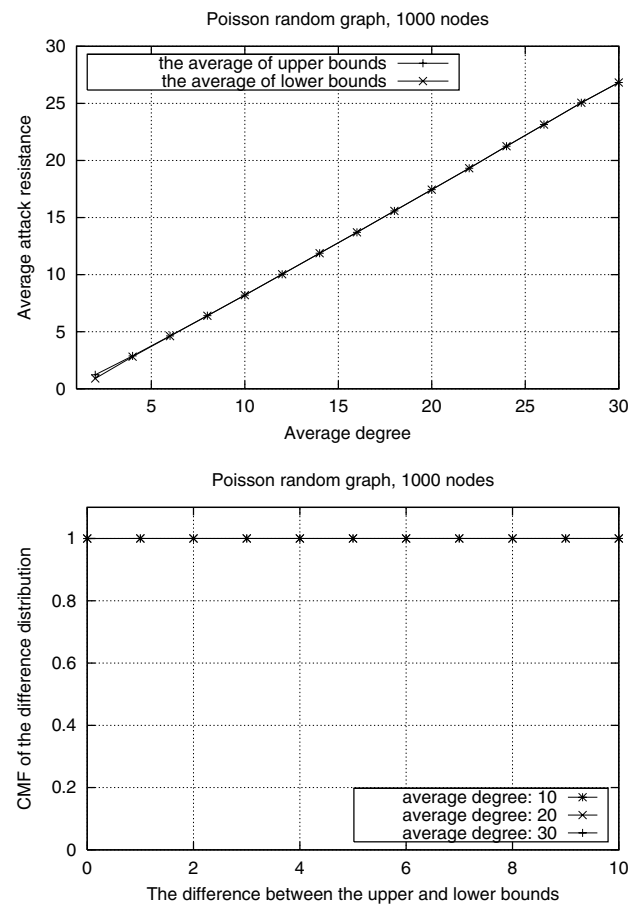

Fig. 1. Experimental results for Poisson random graph: 1000 nodes

Correspondingly, we define $C_{\text {upper }}(G)$ and $C_{\text {lower }}(G)$ as follows:

$$
\begin{aligned}
C_{\text {upper }}(G) & =\frac{1}{N(N-1)} \sum_{u \in V} \sum_{v \neq u \in V} C_{\text {upper }}(u, v), \\
C_{\text {lower }}(G) & =\frac{1}{N(N-1)} \sum_{u \in V} \sum_{v \neq u \in V} C_{\text {lower }}(u, v),
\end{aligned}
$$

which correspond to the average upper bound and the average lower bound of the pairwise connectivity for all pairs of nodes in graph $G$. Obviously, $C_{\text {upper }}(G)$ and $C_{\text {lower }}(G)$ are upper and lower bounds for the APC $C(G)$.

Besides studying the upper bound and lower bound of APC for nodes pairs, we have also studied the distribution of the difference between the upper and lower bounds. Let $d_{\text {diff }}(u, v)$ denote the difference between $C_{\text {upper }}(u, v)$ and $C_{\text {lower }}(u, v)$ :

$$
d_{\text {diff }}(u, v)=C_{\text {upper }}(u, v)-C_{\text {lower }}(u, v) .
$$

Let $d_{\text {diff }}$ denote the random variable for the difference between the upper and lower bounds of any pair of nodes in the network. Then $P\left(d_{d i f f}=k\right)$ can be estimated as follows:

$$
P\left(d_{\text {diff }}=k\right)=\frac{1}{N(N-1)} \sum_{u \in V} \sum_{v \neq u \in V} \mathbf{1}\left[d_{\text {diff }}(u, v)=k\right],
$$

\section{A. Poisson Random Graph}

We first study the APC in Poisson random graph. The experiments are set up as follows: the total number of nodes is $N$, and for any pair of nodes, with probability $p$ there is an edge to directly connect them. It is easy to see that the average node degree is $(N-1) p$. There are two sets experiment $N=1000$ and $N=500$, and in each set, different
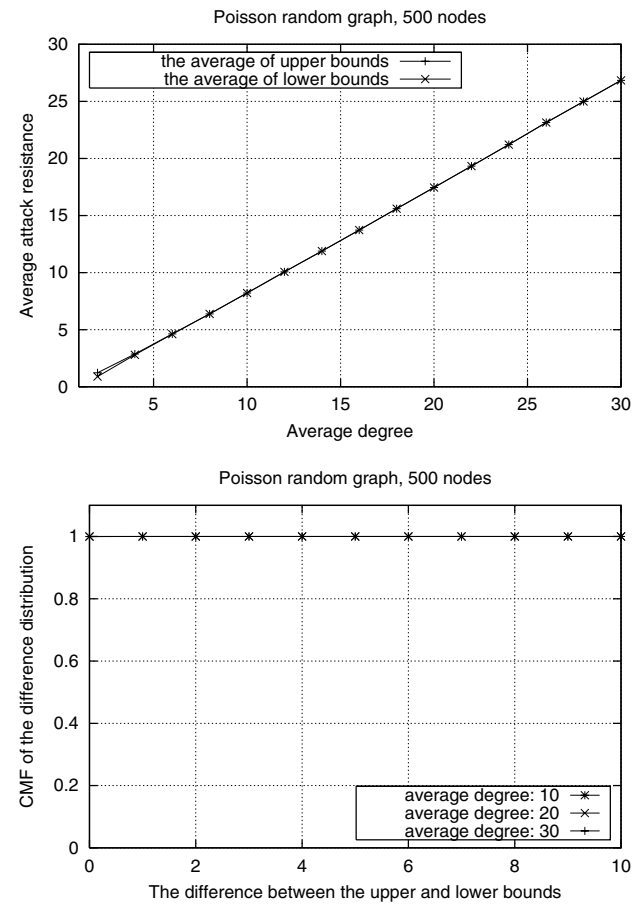

Fig. 2. Experimental results for Poisson random graph: 500 nodes

values of $p$ are tested. The set of experimental results for 1000 nodes are displayed in Fig. 1 and the set of results for 500 nodes are displayed in Fig. 2. Each set of experimental results consists of two subfigures. The first one shows the relationship between the average node degree and the APC for different average node degrees, where both the upper bound $C_{\text {upper }}(G)$ and the lower bound $C_{\text {lower }}(G)$ of the APC are shown. The second one shows the distribution of the difference between the upper and lower bound of the APC under certain average node degree, where three values of average node degree are considered: 10, 20, and 30. For each configuration, the results are averaged over 1000 independently generated Poisson random graphs.

First, from these sets of experimental results we can see that the APC increases with the increase of average node degree, which is easy to understand. Second, it is surprising to see that the upper and lower bounds of the APC almost overlap in all configurations, except when the average node degree is very low (e.g., average node degree is 2 ), which means that the APC of Poisson random graphs can be almost completely characterized by the corresponding upper bound. In another words, in Poisson random graphs, when the total number of nodes is large, the only bottleneck to finding node-disjoint paths between a pair of nodes is the degrees of the two nodes. Third, by comparing the results in Fig. 1 and Fig. 2 we can also see that the APC does not change with the change in the number of nodes in the network.

Now we consider how to get the average upper bound directly using probability theory. In Poisson random graphs, we simply assume that the degree distributions for different nodes are independent, though it is not strictly true. When $N$ 
is large and $(N-1) p=\lambda$, given any pair of nodes $u$ and $v$, it is easy to show that the probability of $C_{u p p e r}(u, v)$ equal to $k$ can be calculated as follows:

$$
\begin{aligned}
& P\left(C_{\text {upper }}(u, v)=k\right) \\
= & P(d(u)=k) P(d(v)>k)+P(d(u) \geq k) P(d(v)=k) \\
= & \frac{e^{-\lambda} \lambda^{k}}{k !}\left(\sum_{i=k+1}^{\infty} \frac{e^{-\lambda} \lambda^{i}}{i !}+\sum_{i=k}^{\infty} \frac{e^{-\lambda} \lambda^{i}}{i !}\right)
\end{aligned}
$$

When $N$ is large, the upper bound of the $\operatorname{APC} C_{\text {upper }}(G)$ is the mean of a large number of random variables with the same distribution given by Equation (9). Under the simplifying assumption that they are independent, according to the Strong Law of Large Numbers, $C_{\text {upper }}(G)$ is approximately equal to $E\left[C_{\text {upper }}(u, v)\right]$, where $E\left[C_{\text {upper }}(u, v)\right]$ can be calculated as following:

$$
\begin{aligned}
& E\left[C_{\text {upper }}(u, v)\right] \\
= & \sum_{k=0}^{\infty} k \frac{e^{-\lambda} \lambda^{k}}{k !}\left(1-\sum_{i=0}^{k} \frac{e^{-\lambda} \lambda^{i}}{i !}+\sum_{i=k}^{\infty} \frac{e^{-\lambda} \lambda^{i}}{i !}\right) \\
= & \lambda-\lambda\left(\sum_{k=0}^{\infty} \frac{e^{-\lambda} \lambda^{k}}{k !} \sum_{i=0}^{k+1} \frac{e^{-\lambda} \lambda^{i}}{i !}-\sum_{k=0}^{\infty} \frac{e^{-\lambda} \lambda^{k}}{k !} \sum_{i=k+1}^{\infty} \frac{e^{-\lambda} \lambda^{i}}{i !}\right) \\
= & \lambda-\lambda \sum_{k=0}^{\infty} \frac{e^{-\lambda} \lambda^{k}}{k !}\left(\frac{e^{-\lambda} \lambda^{k}}{k !}+\frac{e^{-\lambda} \lambda^{k+1}}{(k+1) !}\right)
\end{aligned}
$$

Since there is no closed form for Equation (10), we try to study the relationship between $E\left[C_{\text {upper }}(u, v)\right]$ and $E[d(u)], E[d(v)]$ by truncating the equation at $k=2000$. Fig. 3 illustrates the computed results based on Equation (10), where we let $X=d(u), Y=d(v)$, then $C_{\text {upper }}(u, v)=$ $\min (X, Y)$. The first subfigure shows the ratio between $E\left[C_{\text {upper }}(u, v)\right]$ and the average node degree; it shows that the ratio increases fast when average degree is small, the levels off and approaches 1 asymptotically. The second subfigure shows $E\left[C_{\text {upper }}(u, v)\right]$ for different average node degree; it shows that though the ratio is not constant, $E\left[C_{\text {upper }}(u, v)\right]$ is approximately a linear function of the average node degree. This is consistent with the experimental findings shown in the the top subfigures of Figs. 1 and 2.

$E\left[C_{\text {upper }}(u, v)\right]$ is an unbiased estimator for $C_{\text {upper }}(G)$, i.e., $E\left[C_{\text {upper }}(G)\right]=E\left[C_{\text {upper }}(u, v)\right]$. Thus the mean square error is minimized. The normalized RMSE is shown in Fig. 4 and the RMSE is computed based on 10000 independently generated Poisson random graph. As illustrated, the normalized RMSE is small and decreased when the average node degree is increased, which means $E\left[C_{\text {upper }}(u, v)\right]$ is a good estimator especially when the average node degree is large.

\section{B. Geometric Random Graph}

Now we consider the APC in geometric random graphs. In the experiments, the geometric random graphs are generated as follows: nodes are independently deployed inside a rectangular area of $2000 \mathrm{~m} \times 2000 \mathrm{~m}$ according to the $2 \mathrm{D}$ uniform distribution, and two nodes have a link that directly
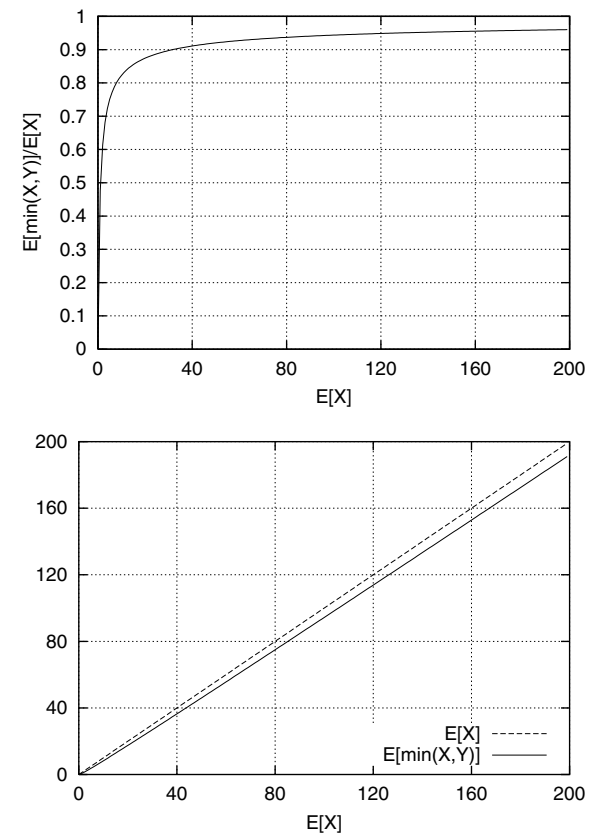

Fig. 3. Relationship between $E\left[C_{\text {upper }}(u, v)\right]$ and $E[d(u)], E[d(v)]$

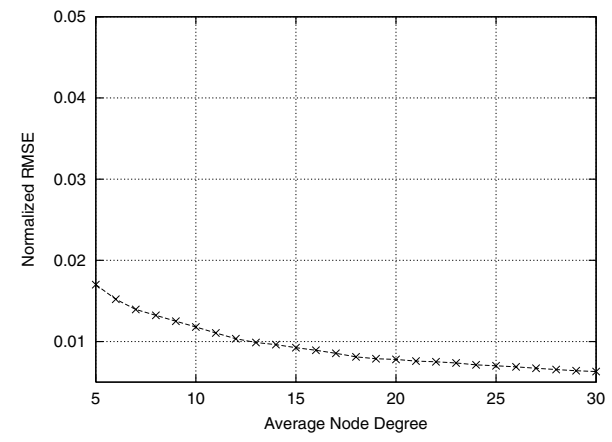

Fig. 4. Relationship between normalized RMSE and average node degree

connects them if and only if the distance between them is no more than $100 \mathrm{~m}$. By changing the total number of nodes, we can generate geometric random graphs with different average node degree. Given the total number of nodes $N$, the average node degree can be approximated as $N \pi / 400$. Similarly, for each set of experiments, two subfigures are generated, and for any specific configuration, the results are averaged over 1000 independently generated geometric random graphs.

Fig. 5 illustrates the results when all node pairs in the network are considered to compute the APC. From this set of results, first we can see that the APC increases with the increase of average node degree, similar to the case of Poisson random graphs. Second, unlike in the case of Poisson random graphs, the upper bound and lower bound of the APC do not overlap. The distribution of the difference between the upper and lower bounds also shows that in many situations the difference can be very large. For example, in all three cases (i.e., average degree equal to 10,20 , or 30 ), with about the probability of $20 \%$ the difference is larger than 4 . One possible reason is that when we perform the experiments, we have not 

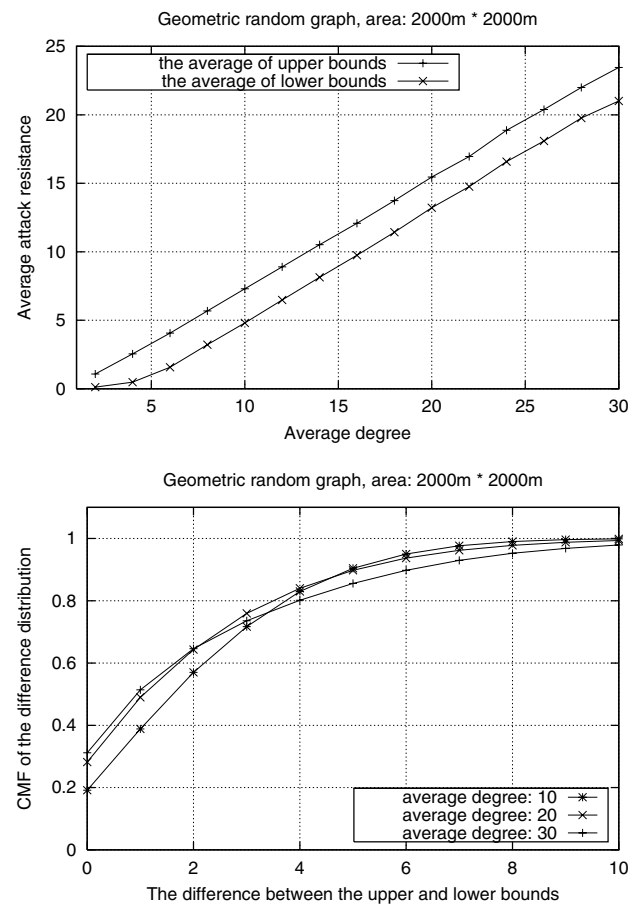

Fig. 5. Experimental results for geometric random graph: all node pairs

considered the boundary effects, while for those nodes near the boundary, they will have much less chance to match their upper bounds due to the asymmetry of the network from the point of view of those nodes.

To investigate the possible boundary effects in geometric random graphs, we have conducted another set of experiments. In this set of experiments, only nodes inside the inner part of the whole area are considered when calculating the APC. Specifically, given a geometric random graph in a rectangular area of $2000 m \times 2000 m$, only node pairs with both of the nodes inside the inner area of $1200 \mathrm{~m} \times 1200 \mathrm{~m}$ are considered, that is, all considered nodes will be at least $400 \mathrm{~m}$ away from the boundary. The experimental results are illustrated in Fig. 6. From the new set of experiment results, first we can see that with the increase of average node degree, the lower bound of the APC becomes closer to the upper bound. Meanwhile from the distribution of the difference between the upper and lower bounds we can also see that the differences between the upper and lower bounds become much smaller. Second, for inner node pairs the value of upper bound for the APC is similar to the case in Poisson random graphs, which makes sense since from the viewpoint of the inner nodes, the geometric random graph is almost same as the Poisson random graph. These results show that when the network is large and the average degree is relatively large, by removing the boundary effects, the upper bound can also approximate very well the APC. Furthermore, the upper bound for the inner nodes pairs can be computed roughly using Equation (10).

\section{CONClusion}

In this paper we have studied the fault tolerance of wireless multihop networks, which are modelled using Poisson random
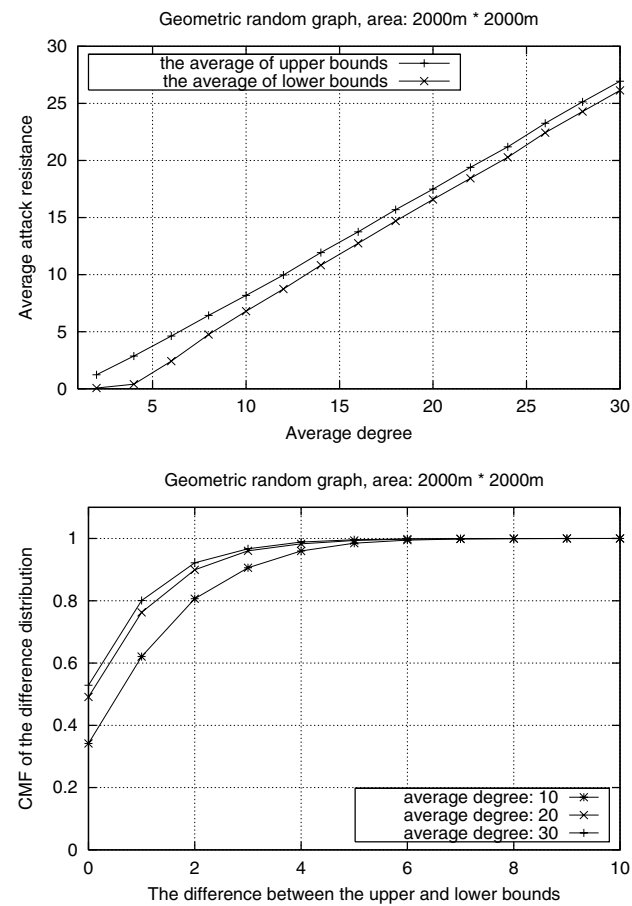

Fig. 6. Experimental results for geometric random graph: nodes pairs inside the inner area of $1200 \mathrm{~m} \times 1200 \mathrm{~m}$.

graphs and geometric random graphs. To focus on expected performance rather than worst case behavior, we have proposed to use a new metric to measure fault tolerance: the average pairwise connectivity.

Experimental studies for the APC are performed in Poisson random graphs and geometric random graphs. The studies have shown that the upper bound of the APC can approximate the APC very well in Poisson random graphs, as well as in geometric random graph when the boundary effects are excluded. We also showed how to estimate the upper bound using probability theory.

\section{REFERENCES}

[1] D. Pradhan, "Dynamically restructurable fault-tolerant processor network architectures," IEEE Transactions on Computers, vol. C-34, pp. 434-447, May 1985.

[2] W. Najjar and J.-L. Gaudiot, "Network resilience: A measure of network fault tolerance," IEEE Transactions on Computers, vol. 39, no. 2, pp. 174-181, February 1990.

[3] C. Bettstetter, "On the minimum node degree and connectivity of a wireless multihop network," in MOBIHOC, 2002.

[4] B. Bollóbas, Modern Graph Theory. Springer, 1998.

[5] P. Erdös and A. R/'enyi, "On random graphs," Publ. Math. Debrecen, vol. 6, pp. 290-297, 1959.

[6] _ " "On the evolution of random graphs," Publ. Math. Inst. Hungar. Acad. Sci., vol. 5, pp. 17-61, 1960.

[7] M. D. Penrose, "On k-connectivity for a geometric random graph," Wiley Random Structures and Algorithms, vol. 15, no. 2, pp. 145-164, 1999.

[8] R. Solomonoff and A. Rapoport, "Connectivity of random nets," Bull. Math. Biophys., vol. 13, pp. 107-117, 1951.

[9] I. Chlamtac and A. Faragó, "A new approach to the design and analysis of peer-to-peer mobile networks," ACM/Baltzer Wireless Networks, vol. 5, Aug 1999.

[10] B. Bollóbas, Random Graphs, 2nd ed. Academic Press, 2001.

[11] M. E. J. Newman, "The structure and function of complex networks," SIAM Review, vol. 45, no. 2, pp. 167-256, 2003. 